\title{
Approaching intermolecular interactions and membrane activity of taxol by FTIR Spectroscopy-implications for anticancer therapeutics
}

\author{
Erhan Süleymanoglu \\ Faculty of Pharmacy, Department of Pharmaceutical Chemistry, Gazi University, Ankara, Turkey; \\ esuleymanoglu@gazi.edu.tr
}

Received 16 October 2009; revised 30 November 2009; accepted 2 December 2009.

\begin{abstract}
We studied the incorporation of hydrophobic drug Taxol into a solid lipid matrices by FTIR spectroscopy. Lipid arrays containing different molar fractions of the drug were made and deposited on the spectrometer glass window substrates for obtaining multilayer stacks. The drug induced an alteration of lipid array spacings, indicating the drug-lipid recognition. Using excess amounts of Taxol provide information on extrapolations on its cellular solubility in biomembranes. The data obtained could be used further for developing novel anticancer drug formulations, as well as for elucidating its novel cellular pharmacological targets.
\end{abstract}

Keywords: Antineoplastic Drugs; Taxol-Membrane Interactions; Structural Changes; FTIR

Spectroscopy

\section{INTRODUCTION}

Taxanes are one of the most widely used antineoplastic agents during the past 25 years. These drugs, which cause cell cycle arrest and apoptosis following microtubule stabilization are currently employed in treatment of common cancers such as breast, lung and ovary $[1,2]$. Since most of the mechanims responsible for their therapeutic action at the molecular level remain to be determined, factors that affect tumour sensitivity, cellular pharmacology and drug disposition have been intensely investigated with the hope that their elucidation could help the development of taxanes with improved efficacy, boavailability and pharmacokinetics.

The various ways of administration of these lipophilic drugs is often problematic due to their low aqueous solubility. Use of solubilisers and other formulations with a high dissolution rate therefore becomes a necessity in order to achieve their therapeutic dose. Phospholipids can be used to solubilise such drugs, with spontaneous formation of complexes. Thus, encapsulation of hydrophilic and binding of amphipathic as well as lipophilic drugs is possible. Studying such drug-lipid recognitions is important also in terms of aquiring detailed knowledge of cellular functions of biomembranes and developing novel models for better understanding of drug action [3].

Pharmaceutical and physicochemical analyses of drugmembrane complexes have been studied by various analytical methods employing a variety of drugs and model membranes. Thermodynamic, spectroscopic and microscopy approaches were used for structural characteization of these macromolecular associations. Among these, vibrational spectroscopy (Infrared and Raman spectroscopy) provide useful information on dynamic changes occuring after complex formation of various biomolecules [4], making it a preferred method of analysis which deserves to be employed also in drugmembane studies. In its most popular mode of application, the infrared (IR) measurements involve water removal from samples, which often give rise to erraneous interpretations of the resulting spectra. Undoubtedly, understanding the interactions of drugs with biomembrane or with liposomal lipids following vesicle encapsulation would help to develop improved therapeutic formulations. Obviously, these are accompanied by release or uptake of ions or water molecules. Determination of hydration/re-hydration effects on the physicochemical characteristics of entrapped drugs depends on the evaluation of the amount and structure of the bound water. In addition, a variety of the existing polymorphs and their different efficiencies is worth studying in more detail. The structural transitions of Taxol-a widely used chemotherapeutic drug and phospholipids following their recognition and complex formation is briefly reported herein. The objective is to relate and use 
the obtained data further in pharmaceutical profiling and design of lipid based anticancer drug delivery systems.

\section{MATERIALS AND METHODS}

Materials: L- $\alpha$-phosphatidylcholine was purchased from Avanti Polar Lipids (Alabaster, AL, USA). KBr and Taxol were a product of Sigma Chem. Co., MI (USA).

FTIR Spectroscopy: Infrared spectra were recorded on IFS 66/S FTIR spectrometer (Bruker Analytische Messtechnik GmbH, Karlsruhe, Germany), equipped with $\mathrm{He}-\mathrm{Ne}$ laser detector and $\mathrm{KBr}$ beam splitter. $\mathrm{KBr}$ pellet method was employed as FTIR sampling technique. The samples were prepared by mixing of lipid and drug by mass in the desired ratios and pressing the mixtures with a die press. Spectra were collected after short incubation of lipid with Taxol. Interferograms were accumulated over the spectral range $4000 \mathrm{~cm}^{-1}$ to $400 \mathrm{~cm}^{-1}$, with a nominal resolution of $2 \mathrm{~cm}^{-1}$ and a minimum of 320 scans. The criterion for elimination of water effect from the spectra was based on the stright baseline between $1750 \mathrm{~cm}^{-1}$ and $2200 \mathrm{~cm}^{-1}$, where the water combination mode is located.

\section{RESULTS AND DISCUSSION}

In the present work, Taxol-lipid mixtures in different ratios were deposited on the FTIR slides to form oriented stacks. The intention was to correlate solid-state structural parameters, especially when higher drug ratios are used and to make further deductions regarding the application of FTIR spectroscopy in this mode for determination of Taxol organization in biomembranes of both normal an malignant cells.

Such an information on the physical states of drugs entrapped in lipidic surrounding simulating cellular interfaces is very useful for predicting and designing improved formulation properties. For better understanding of how Taxol recognizes and binds to cell surface lipids and how it affects the packing and fluidity of membranes, it is necessary to study its interactions with phospholipid molecules emphasizing the engaged functional groups. Since drugs exert their cellular effects in a concentration dependent maner, it is useful to study the effect of various Taxol concentrations on a simulated membrane interface. This would give further insights into the significance of surface activity of drug action. Thus, in this study, the excess drug effects were followed by varying drug concentration and keeping lipid concentration fixed. This would give further details concerning the concentration dependent Taxol effects on membranes and more interestingly would depict the issue of possible mechanisms of drug-induced mem- brane deformation. Such a design is based on the fact that the addition of a drug induces certain perturbations or leads to microdomain formation of phospholipid moieties, which is detectable by FTIR spectroscopic measurements.

Figure 1 shows the effect of increasing drug ratios on the structure of the lipid. The IR spectra are highly sensitive to existance of drug molecule in concentration dependent fashion. In its lowest concentration Taxol restricts the phospholipid flexibility. The highly ordered all-trans hydrocarbon phospholipid chain predominated. Gauche-(kinked) conformation, which leads to higher rotational freedom was not depicted. Used in higher amounts (Figure 1), the drug increases its affinity of binding to phospholipid stacks. Thus, in higher amounts, Taxol fluidized the phospholipid dispersion, as deduced also from the subsequent thermal behaviour of the relevant drug-lipid binary mixtures. These observations are explained by the chemical structure of the employed egg phosphatidylcholine and from the spatial features of the drug molecule. As a bulky molecule, Taxol cannot reach the inner parts of the hydrated phospholipid hydrocarbon chains. It only bound hydrophobic parts such as their acyl chains. Under these conditions, the amorphous Taxol converted to anhydrous state, suggesting that several solid-state structures of the drug coexist, in agreement with [5]. In their elegant approach, J. H. Lee et al. [6] emphasized the importance of studying the various solid properties of Taxol. Major lipid specific band frequencies were seen when equimolar ratios of drug-lipid complexes were used. The most important of these were those belonging to $\mathrm{CH}$ vibrations (2750-3100 $\mathrm{cm}^{-1}$ ) and $\mathrm{CH}_{2}$ wagging progression, as well as $\mathrm{CH}_{2}$ scissoring vibrations within the region $1150-1400 \mathrm{~cm}^{-1}$ and $1465-1475 \mathrm{~cm}^{-1}$. The former indicates the conformational order while the later two provide information about packing effects of the acyl chains, respectively. $\mathrm{PO}_{2}^{-}$vibrations are located at $1242 \mathrm{~cm}^{-1}$. On the other hand, Taxol showed substantial bands at 1600-1750, $1180-1300$ and $630-770 \mathrm{~cm}^{-1}$. Besides small shifts, all of them were seen in the spectra of various drug-lipid mixtures, depicting a drug dominated spectrum, indicating their recognition and binding. Upon comparison with the spectra of the unbound drug and lipid shifts of Taxol's carbonyls from $1736 \mathrm{~cm}^{-1}$ to $1732 \mathrm{~cm}^{-1}$ and from 1736 $\mathrm{cm}^{-1}$ to $1707 \mathrm{~cm}^{-1}$ were seen. These were retained also in higher drug ratios. The strong bands at $3300-3500 \mathrm{~cm}^{-1}$ were due to $\mathrm{OH}$-groups linked to the $\mathrm{H}$-bonds. Following drug-lipid recognition, the intensities were diminished and additional band shifts occured. Apparently, this indicates a conformational restriction of lipid due to bond formation with the drug, depicting the decrease in drug flexibility. In conclusion, FTIR spectroscopy clearly 


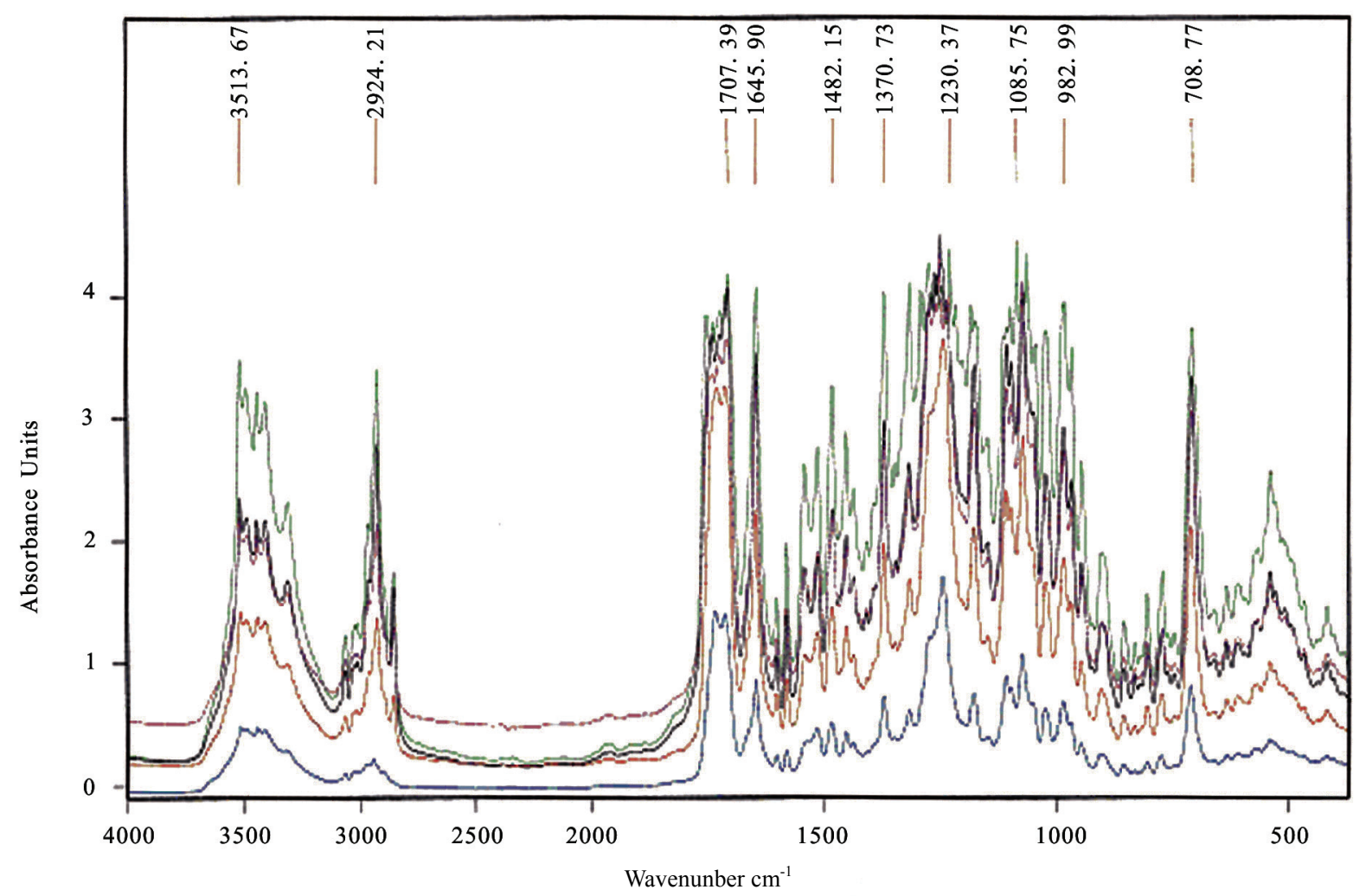

Figure 1. Overall infrared spectra of various mixtures of Taxol with L- $\alpha$-phosphatidylcholine. Samples were prepared as described in Materials and Methods section. The effect of excess drug ratios are shown from bottom to top: $1: 1 ; 1: 3 ; 1: 5 ; 1: 7$ and $1: 10$, lipid/drug fractions, respectively.

showed that Taxol-lipid recognition and binding was governed by $\mathrm{CH}$ and $\mathrm{CH}_{2}$ groups, as well as $\mathrm{H}$-bonded surface $\mathrm{OH}$-groups. $\mathrm{PO}_{2}^{-}, \mathrm{C}=\mathrm{O}, \mathrm{C}=\mathrm{C}$ and $\mathrm{CH}_{2}$ groups of the drug were also engaged.

Thus, by following such a vibrational spectroscopic approach, further insights into Taxol-membrane interactions could be obtained and used for the understanding of the pharmacological activities of its many formulations. In this respect, determining its recognition with biomembranes employing dried cellular lipids becomes crucial. Structural studies of Taxol-lipid arrangements, by FTIR spectroscopy, as presented, can give valuable information about the orgnization of the drug in cell membranes and can help to optimize lipid matrix concerning its solubility potential. On the other hand, the issue of how higher amounts of the drug lead to membrane deformation could be clarified. For instance, a novel hypothesis can be generated concerning the item that rather than being driven only by the formation of membrane-associated structural scaffolds, drug-induced membrane deformation may require physical perturbation of the lipid bilayer [7]. An emerging theme in this process would be the importance of lipophilic drugs that partially penetrate the lipid bilayer. In addition, such an IR spectrosopic approach will define further important issues, such as the particular mechanisms engaged in the binding of lipophilic drugs with liposomal membranes; the reversibility of this binding; the role played by other factors in these recognitions with liposomal membranes and release kinetics after entrappment, e.g. the significance of the biological mileu or the involvement and specificity of other lipid membranes.

Cell membranes have always been regarded as an important subcellular organelle, both as a site of pathogenesis and as a therapeutic target. The issue of the use of lipids as targets for overcoming antineoplastic drug resistance is very interesting to study. How tumor cell membrane alterations influence the response of cancer to chemotherapy still remains to be defined. Exciting future directions of studying Taxol-lipid interactions exist regarding novel cancer treatment strategies. The motivation for starting research in this field has come from recent data on the role of lipid phase of cell membranes in P-gp-mediated MDR activity, as well as in reversal of tumor resistance to apoptotic stimuli. To prove the possible Pgp-independent pathway of action causing chemoresistance and increasing membrane permeability, various Taxol-biomembrane systems involving molecules leading to alterations in membrane fluidity and to increase in chemosensitivity (chemosensitizers) could be 
employed in a dose-dependent manner. This could be followed by applying fluorescence measurements in isolated tumor biomembranes, in model membrane systems or in wild type or MDR animal and human cell membranes. Thus, studying membrane dynamics in both the outer hydrophobic region of the bilayer and in the acyl chain region, respectively would be possible. Using such chemosensitizers acting by increasing lipid bilayer permeability would correlate with anticancer cytotoxicity. Thus, a new model can be build, based on the action of chemosentisizers causing changes in membrane structures and leading to membrane fluidity fluctuations and increasing bilayer permeability, showing the significant role of these events in Taxol cytotoxicity. Subsequently, new therapeutic strategies can be designed.

Unfortunately, the conventional drug development strategies suffer from high rates of design drawbacks due to inability to predict tumor responce at an early stage of the treatment. Thus, the employed therapy regimes can not be individualized in terms of personalized medicine. On the other hand, the currently used laboratory medicine protocols often lead to high numbers of false-negative results. Hence, there is a need for development of novel methods both for detecting malignancy at an early stage, as well as for predicting tumor responce to drug therapy. Spectroscopy offers new possibilities in this respect. In contrast to conventional histological techniques, vibrational spectroscopic approaches, for instance, do not require a special sample preparation. Undoubtedly, with the current achievements of designs of light sources, optical components, detectors and algorithms for data processing, often employing artificial intelligence prorammes such as neural network computing, the number of their applications in disease recognition will increase in the near future. The unappreciated yet biotherapeutic potential of these methods in designing new cancer therapy schemes should be emphasized. Vibrational spectra of cells, tissues and biological fluids are a reflection of total cellular chemistry and structure. Therefore, they provide a measure for the entire chemical cellular status regarding such metabolic events as cell cycle events, differentiation, growth and cell death. Hence, vibrational spectroscopy provide important information on vital cellular activities, e.g. transcriptional and translational regulations, as well as on post-translational modifi cations. Therefore, there is increasing interest in the biomedical field in using these methods as emerging biophotonic tools for diagnostic in situ and in vivo therapeutic applications. The idea here, is to appreciate that human pathologies are accompanied by alterations in the chemical compositions of cells, tissues, organs, or body fluids. In this context, vibrational spectroscopy, such as FTIR methods, appear to be ideally suited for sensitive detection of such changes of the secondary structures of the engaged biomacromolecules as a diagnostic technique. Biomedical IR spectroscopy probes biological samples in a way that the active vibrational modes of all constituents present in the mixture are followed in a single experiment, resulting in a very complex spectra though entire spectral range. Thus, the obtained spectra provide spectral fingerprints of the total chemical composition of the biosample under study. Applied to cellular systems, such measurements are useful for gaining further information on cellular chemical structures, which are potential targets for novel anticancer drug designs. Therefore, by following a tumor specific biomolecular dynamics, new disease specific drug designs can be suggested. For instance, proper knowledge of the secondary structural changes of the nucleic acid topologies and their role in cellular transcriptional and translational machinery of a tumor cell is a pre-requisite for designing efficient sequence specific DNA binding anticancer agents. In our opinion, especially when coupled to microscopic systems, IR and Raman spectroscopic techniques will represent important tools improving the efficacy of the standard laboratory methods for disease recognition and therapy.

\section{ACKNOWLEDGEMENTS}

This work was supported by Gazi University Research Foundation (Project No: 02-2005/15). The technical assistance of Dr. N. Özkâr and Dr. N. Özkan from The Central Laboratory of Middle East Technical University (Ankara-Turkey) is greatly acknowledged.

\section{REFERENCES}

[1] Hennenfent, K.L. and Govindan, R. (2006) Novel formulations of taxanes: A review. Old wine in a new bottle? Annals of Oncology, 17(5), 735-749.

[2] Kruh, G.D. (2005) Ins and outs of taxanes. Cancer Biology \&Therapy, 4(9), 1030-1032.

[3] Seydel, J.K. and Wiese, M. (2002) Drug-membrane interactions, analysis, drug distribution, modeling. In Mannhold, R., Kubinyi, H. and Folkers, G., Eds., Methods and Principles in Medicinal Chemistry, Wiley-VCH Verlag GmbH, Weinheim, 15.

[4] Günzler, H. and Gremlich, H.-U. (2002) IR spectroscopy. An introduction. Wiley-VCH Verlag $\mathrm{GmbH}$, Weinheim, 69469.

[5] Liggins, R.T., Hunter, W.L. and Burt, H.M. (1997) Solidstate characterization of paclitaxel. Journal of Pharmaceutical Sciences, 86(12), 1458-1463.

[6] Lee, J.H., Gi, U.-S., Kim, J.-H., Kim, Y., Kim, S.-H., Oh, H. and Min, B. (2001) Preparation and characterization of solvent induced dihydrated, anhydrous, and amorphous Paclitaxel. Bulletin of the Korean Chemical Society, 22(4), 925-928.

[7] Farsad K. and De Camilli, P. (2003) Mechanisms of membrane deformation. Current Opinion in Cell Biology, 15(4), 372-381. 